\title{
Long flexible polymers interacting with ellipsoids, cylinders, and needles
}

\author{
E. Eisenriegler ${ }^{\mathrm{a})}$ \\ Institut für Festkörperforschung, Forschungszentrum Jülich, D-52425 Jülich, Germany
}

(Received 3 October 2006; accepted 17 October 2006; published online 22 November 2006)

\begin{abstract}
The depletion interactions of ellipsoidal colloidal particles in a solution of long polymer chains are analyzed. Of primary concern are the limiting cases in which the ellipsoid reduces to a cylinder of infinite length and finite radius and a "needle" of finite length and vanishing radius. Relations are obtained between the polymer effects induced by a needle that is much shorter than the polymer size and by a cylinder with radius much smaller than the polymer size. These imply exact quantitative results for the orientation-dependent depletion interaction between a short needle and a wall. Qualitative differences between the needle and thin disk are discussed. (C) 2006 American Institute of Physics. [DOI: 10.1063/1.2393229]
\end{abstract}

\section{INTRODUCTION}

Colloidal particles immersed in a solution of nonadsorbing polymer chains experience an effective interaction. ${ }^{1,2}$ It is induced by polymer depletion near the particle surfaces and depends on the density and molecular weight of the chains, the sizes and shapes of the particles, and their mutual distances and orientations. ${ }^{3}$ For mesoscopic particles and long flexible chains it is universal, i.e., independent of the chain microstructure. Pure polymer systems also have universal properties. ${ }^{4}$ For example, both the Flory exponent $\nu$ describing the dependence on molecular weight $M$ of the mean square end-to-end distance $\mathcal{R}^{2} \equiv d \mathcal{R}_{x}^{2} \propto M^{2 \nu}$ and the scaling dimension of the monomer density,

$$
m=d-\frac{1}{\nu}
$$

in the density-density correlation function are universal. Here $d$ is the spatial dimension. For self-avoiding chains in $d=3, \nu=0.588$ and $m=1.30$. For ideal chains without selfavoidance, $m=d-2$. The interaction between chain monomers and the particle surface also has universal features and can be modeled by a hard repulsion, in general.

Consider colloidal particles with the shapes of ellipsoids with an axis of rotation symmetry and with large and small diameters $l$ and $s$, respectively. The large diameter coincides with the rotation axis for prolate ellipsoids and is perpendicular to it for oblate ellipsoids. There are important special cases: the sphere if $s / l=1$, the needle or thin circular plate ("disk") if $s / l \ll 1$ with $l$ finite for a prolate or oblate ellipsoid, and the infinitely extended cylinder and plate if $s / l$ $\rightarrow 0$ with $s$ finite.

To what extent is the interaction of polymers with a needle related to the interaction with an infinitely long $c y l$ inder? For a long needle with $l \gg \mathcal{R}_{x}$, the relation is obvious since a polymer chain senses the needle as a cylinder with the local radius. This applies not only to a long thick needle with $\mathcal{R}_{x}<s \ll l$ but even to a long thin needle with $s \ll \mathcal{R}_{x}$

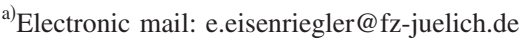

$\ll l$, which is related to a thin cylinder with radius $R$ much smaller than $\mathcal{R}_{x}$.

As discussed below, the perturbation of self-avoiding polymers due to a thin cylinder in $d=3$ dimensions is proportional $^{5,6}$ to $R^{m-1}=R^{0.30}$, which vanishes as $R$ tends to zero. This is very different from a thin infinite plate where the perturbation remains finite if the width of the plate tends to zero. ${ }^{7}$

Our main interest is in small mesoscopic needles with $s \ll l \ll \mathcal{R}_{x}$. While less obvious in this "nanoparticle" case, we show that the leading isotropic and orientation-dependent polymer effects induced by a small needle can also be derived from the effects induced by a thin cylinder. This applies even when distant perturbations act upon the polymerparticle system. As an example, we predict the orientationdependent polymer-induced interaction between the needle and a wall from polymer properties near a thin cylinder without a wall and in the half space without a particle. We also point out the differences between ideal ${ }^{8}$ and self-avoiding polymers as depletants.

In order to compare the needle with a disk or with more compact anisotropic particle shapes, we also consider nanoparticle ellipsoids with arbitrary aspect ratios $s / l$. The induced polymer effects can be expanded in powers of the

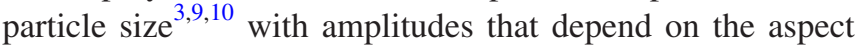
ratio and become singular in the needle limit. For example, the leading perturbation due to a small particle is isotropic and proportional to the particle size raised to the power $m$, since it arises from coupling to the monomer density. ${ }^{3,9}$ In the needle limit and for self-avoiding chains, its amplitude acquires the power law dependence $(s / l)^{m-1}$, consistent with the plausible result that the leading, isotropic perturbation strength of the needle is proportional to $l s^{m-1}$. Similar but less obvious power laws and their universal amplitudes will be derived for the orientation-dependent polymer effects of the needle.

These exact results are obtained in Sec. II by comparing the polymer Boltzmann weights arising from a small needle and a thin cylinder which can be expanded in terms of point operators $^{3}$ and line integrals of operators, ${ }^{5,11}$ respectively. 
While self-avoiding chains in $d=3$ are of primary interest, it is instructive to check the two expansions and the relations between them for ideal polymers in $d>3$, where $m-1$ is also positive. This is done in Sec. III where we discuss the interactions of cylinders and prolate ellipsoids of arbitrary aspect ratio with ideal polymers in $d>3$. The results are needed in order to extend the analysis to self-avoiding polymers by means of an $\varepsilon=4-d$ expansion.

\section{SMALL ELLIPSOIDS AND THIN CYLINDERS}

There is a "polymer-magnet" correspondence ${ }^{4}$ between a nonadsorbing polymer interacting with a colloidal particle and an $n$-vector field theory outside a particle with an "ordinary" surface. ${ }^{12}$ The Boltzmann factor of a small ellipsoidal particle at $\mathbf{r}_{P}$ in the field theory can be expanded as ${ }^{10}$

$$
\begin{aligned}
\exp \left(-\delta \mathcal{H}_{\mathrm{ell}}\right) \propto & 1-\mathcal{I} \psi\left(\mathbf{r}_{P}\right)-\mathcal{N} T_{\|||}\left(\mathbf{r}_{P}\right)-\mathcal{N}^{\prime} \partial_{\|}^{2} \psi\left(\mathbf{r}_{P}\right) \\
& -\cdots,
\end{aligned}
$$

while for a particle with the form of a thin, infinitely long cylinder ${ }^{5,11}$

$$
\begin{aligned}
\exp \left(-\delta \mathcal{H}_{\mathrm{cyl}}\right) \propto & 1-\mathcal{J} \int_{-\infty}^{\infty} \mathrm{d} r_{1 \|} \psi\left(r_{1 \|}, \mathbf{0}\right) \\
& -\mathcal{K} \int_{-\infty}^{\infty} \mathrm{d} r_{1 \|} \int_{-\infty}^{\infty} \mathrm{d} r_{2 \|} \psi\left(r_{1 \|}, \mathbf{0}\right) \psi\left(r_{2 \|}, \mathbf{0}\right) \\
& -\cdots
\end{aligned}
$$

Here $^{10}$ the leading isotropic operator $\psi$ equals $-\mathcal{A}_{\epsilon} \epsilon / B_{\epsilon}^{1 / 2}$, where $\epsilon$ is the energy density of the field theory with scaling dimension $x_{\epsilon}$, and $B_{\epsilon}$ and $\mathcal{A}_{\epsilon}$ are the amplitudes of the bulk two-point function $\langle\epsilon(\mathbf{r}) \epsilon(\mathbf{0})\rangle_{\text {bulk }}=B_{\epsilon} r^{-2 x_{\epsilon}}$ and the half space ("hs") profile $\left\langle\epsilon\left(\mathbf{r}_{p}, z\right)\right\rangle_{\mathrm{hs}} / B_{\epsilon}^{1 / 2}=\mathcal{A}_{\epsilon} z^{-x_{\epsilon}}$ at the critical point. In the half space, $\mathbf{r}_{p}$ and $z$ denote the components of $\mathbf{r}$ parallel and perpendicular to the planar boundary. The subscript \| denotes the direction parallel to the ellipsoid or cylinder axes. The anisotropic operators in (2.1) are the diagonal component parallel to the axis $T_{\|\|}$of the stress tensor of the field theory and the second derivative $\partial_{\|}^{2} \psi$ of $\psi$ parallel to the axis. ${ }^{13}$ The scaling dimension of $T_{\|\|}$equals the spatial dimension $d$. In the cylinder expansion (2.2), the components $r_{\|}$and $\mathbf{r}_{\perp}$ of $\mathbf{r}$ are parallel and perpendicular to the cylinder axis, $\psi\left(r_{\|}, \mathbf{0}\right)$ means $\psi\left(r_{\|}, \mathbf{r}_{\perp}=\mathbf{0}\right)$, and the double integral is understood to be dimensionally regularized at $r_{1 \|}=r_{2 \|}$ when inserted in correlation functions.

In the asymptotic scaling region, ${ }^{14} \mathcal{I} \rightarrow l^{x} \epsilon \hat{I}, \mathcal{N} \rightarrow l^{d} \hat{N}$, and $\mathcal{N}^{\prime} \rightarrow l^{x} \epsilon^{+2} \hat{N}^{\prime}$, where $\hat{I}, \hat{N}$, and $\hat{N}^{\prime}$ are universal functions of $s / l$, and $\mathcal{J} \rightarrow R^{x_{\epsilon}-1} J$ and $\mathcal{K} \rightarrow R^{2\left(x_{\epsilon}-1\right)} K$, with universal ${ }^{15}$ numbers $J$ and $K$, since the Boltzmann weight has vanishing scaling dimension. In the polymer ${ }^{4}$ limit $n \rightarrow 0$,

$$
x_{\epsilon} \rightarrow m,
$$

where $m$ is the scaling dimension of the monomer density mentioned in the Introduction, and $\psi$ corresponds to the monomer-density operator. Compare Eqs. (2.8)-(2.10) in Ref. 10.

As explained in more detail in Refs. 3, 5, and 10, operator expansions in the field theory such as (2.1) and (2.2) allow one to calculate polymer properties in the presence of small ellipsoids and thin cylinders. They are determined by means of Laplace transforms of correlation functions of the $n \rightarrow 0$ field theory, with the molecular weight of the polymer chain and the deviation $t$ from the critical temperature of the field theory as Laplace conjugates. ${ }^{4}$ The expansions are analogous to the well known operator product expansions in field theory, and their amplitudes do not depend on the presence of distant perturbations. Moreover the leading amplitudes $\mathcal{I}, \mathcal{N}$, and $\mathcal{N}^{\prime}$ (or $\mathcal{J}$ and $\mathcal{K}$ ) are independent of $t$. Thus they can be determined by investigating the field theory right at the critical point with a single embedded ellipsoid (or cylinder) and then used to study the effective interaction between several particles (or a particle and a wall) induced by long polymers and the dependence on their molecular weight.

In case of the needle the small particle amplitudes $\mathcal{I}, \mathcal{N}$, and $\mathcal{N}^{\prime}$ are related to the cylinder amplitudes $J$ and $K$. On generalizing the cylinder expansion (2.2) to the case where the cylinder diameter is slowly varying along the axis, i.e., to $2 R \rightarrow s \sigma(\varrho)$ with $\varrho=r_{\|} /(l / 2)$, the dominant terms are again given by (2.2) with $\mathcal{J}$ and $\mathcal{K}$ put inside the integrals and replaced by the local expressions ${ }^{16}$

$$
\mathcal{J}=J R^{x} \epsilon^{-1} \rightarrow J\left[(s / 2) \sigma\left(\varrho_{1}\right)\right]^{x} \epsilon^{-1}
$$

and

$$
\mathcal{K}=K R^{2\left(x_{\epsilon}-1\right)} \rightarrow K(s / 2)^{2\left(x_{\epsilon}-1\right)}\left[\sigma\left(\varrho_{1}\right) \sigma\left(\varrho_{2}\right)\right]^{x} \epsilon^{-1} .
$$

The $r_{\|}$integrations are now confined to the interval $-l / 2$ $\leqslant r_{\|} \leqslant l / 2$, and

$$
\sigma(\varrho)=\left(1-\varrho^{2}\right)^{1 / 2}
$$

for an ellipsoidal needle with center at $\mathbf{r}_{P}=(0, \mathbf{0})$. In the small particle case, one considers distances from the needle center much larger than $l$ and expands the operator

$$
\psi\left(\frac{l}{2} \varrho_{1}, \mathbf{0}\right)=\psi(0, \mathbf{0})+\frac{1}{2}\left(\frac{l}{2}\right)^{2} \varrho_{1}^{2} \partial_{\|}^{2} \psi(0, \mathbf{0})+\cdots
$$

and the operator product

$$
\begin{aligned}
\psi\left(\frac{l}{2} \varrho_{1}, \mathbf{0}\right) \psi\left(\frac{l}{2} \varrho_{2}, \mathbf{0}\right) \rightarrow & \frac{C_{\psi \psi T}}{B_{T}}\left(\frac{l}{2}\left|\varrho_{1}-\varrho_{2}\right|\right)^{d-2 x_{\epsilon}} T_{\|\|}(0, \mathbf{0}) \\
& +\cdots
\end{aligned}
$$

inside the single and double integral. In (2.7) a first derivative has been omitted, since it does not contribute to the integral $\int \mathrm{d} r_{\|}$for a symmetric $\sigma$. To obtain (2.8), we have used the operator product expansion, where $C_{\psi \psi \psi}$ and $B_{T}$ are universal amplitudes of the bulk three-point function $\left\langle\psi \psi T_{\|\|}\right\rangle_{\text {bulk }}$ and the two-point function $\left\langle T_{k l} T_{m n}\right\rangle_{\text {bulk. For ex- }}$ plicit definitions see Eqs. (2.39), (2.17), and (A9) of Ref. 17. There are also terms proportional to $\psi(0, \mathbf{0})$ and $\partial_{\|}^{2} \psi(0, \mathbf{0})$ on the right hand side of (2.8), but their contribution to $\mathcal{I}$ and $\mathcal{N}^{\prime}$ is smaller by a factor of $(s / l)^{x} \epsilon^{-1}$ than the contribution from (2.7) and they do not contribute to $\mathcal{N}$.

Inserting (2.4)-(2.8) in (2.2) and comparing with (2.1), one finds for the needle 


$$
\left(\mathcal{I}, \mathcal{N}^{\prime}\right) \rightarrow J\left(\frac{s}{2}\right)^{x_{\epsilon}-1} \frac{l}{2} \int_{-1}^{1} \mathrm{~d} \varrho[\sigma(\varrho)]^{x_{\epsilon}-1}\left(1, \frac{1}{2}\left(\frac{l}{2}\right)^{2} \varrho^{2}\right)
$$

and

$$
\begin{aligned}
\mathcal{N} \rightarrow & K\left(\frac{l}{2}\right)^{d}\left(\frac{s}{l}\right)^{2\left(x_{\epsilon}-1\right)} \frac{C_{\psi \psi T}}{B_{T}} \\
& \times \int_{-1}^{1} \mathrm{~d} \varrho_{1} \int_{-1}^{1} \mathrm{~d} \varrho_{2}\left[\sigma\left(\varrho_{1}\right) \sigma\left(\varrho_{2}\right)\right]^{x_{\epsilon}-1}\left|\varrho_{1}-\varrho_{2}\right|^{d-2 x} \epsilon .
\end{aligned}
$$

\section{IDEAL POLYMERS INTERACTING WITH ELLIPSOIDS AND CYLINDERS}

An ideal chain without self-avoidance corresponds to a field theory without anharmonicities. The $n$-component field theory decouples into $n$ independent one-component Gaussian models, the scaling dimension $x_{\epsilon}=m=d-2$, and the amplitudes $\mathcal{I}, \mathcal{N}$, and $\mathcal{N}^{\prime}$ and $\mathcal{J}$ and $\mathcal{K}$ are independent of $n$. We consider the partition function $Z\left(L ; \mathbf{r}, \mathbf{r}^{\prime}\right)$ of a polymer with the two ends fixed at $\mathbf{r}$ and $\mathbf{r}^{\prime}$, its Laplace transform

$$
\int_{0}^{\infty} \mathrm{d} L \exp (-L t) Z\left(L ; \mathbf{r}, \mathbf{r}^{\prime}\right)=G\left(t ; \mathbf{r}, \mathbf{r}^{\prime}\right) \equiv\langle\varphi\rangle,
$$

which is the propagator of the one-component Gaussian model with Hamiltonian $H=\int d \mathbf{r}^{\prime \prime}\left[(\nabla \Phi)^{2}+t \Phi^{2}\right] / 2$ and Dirichlet $^{3,4}$ boundary conditions $\Phi=0$ on the surface of embedded particles, and the density profile of polymer ends

$$
\mathcal{E}(L, \mathbf{r})=\int \mathrm{d} \mathbf{r}^{\prime} Z\left(L ; \mathbf{r}, \mathbf{r}^{\prime}\right) .
$$

Here we have used the notation $\varphi=\Phi(\mathbf{r}) \Phi\left(\mathbf{r}^{\prime}\right)$, and $L$ $=\mathcal{R}_{x}^{2} / 2$ characterizes the molecular weight of the polymer chain. The partition function and end density obey ${ }^{3,4}$ the equations

$$
\left(\Delta_{\mathbf{r}}-t\right) G\left(t ; \mathbf{r}, \mathbf{r}^{\prime}\right)=-\delta\left(\mathbf{r}-\mathbf{r}^{\prime}\right)
$$

and

$$
\left(\Delta_{\mathbf{r}}-\partial_{L}\right) \mathcal{E}(L, \mathbf{r})=0 .
$$

We consider the polymer system in $d$ dimensions with a single embedded ellipsoid or cylinder. The space perpendicular to the particle axes is thus $d-1$ dimensional. As mentioned in the Introduction our main focus is ${ }^{6,8}$ on $d>3$, where $x_{\epsilon}-1=d-3$ is positive and the cylinder expansion (2.2) makes sense. We shall discuss, in particular,

$$
e(\mathbf{r})=[\mathcal{E}(L, \mathbf{r})]_{L=\infty}
$$

and

$$
g\left(\mathbf{r}, \mathbf{r}^{\prime}\right)=\left[G\left(t ; \mathbf{r}, \mathbf{r}^{\prime}\right)\right]_{t=0}
$$

which obey $\Delta e=0$ and $\Delta g=-\delta\left(\mathbf{r}-\mathbf{r}^{\prime}\right)$. The results allow us to test the expansions (2.1) and (2.2) and yield the relations (2.9) and (2.10) for the needle geometry. The ideal chain universal amplitudes $\mathcal{I}, \mathcal{N}$, and $\mathcal{N}^{\prime}$ that we calculate for arbitrary aspect ratio $s / l$ and $d$ can be used as the starting point in an $\varepsilon=4-d$ expansion of self-avoiding chain properties.

\section{A. Prolate ellipsoid}

Consider a prolate ellipsoid in a $d$-dimensional space $\mathbf{r}$ $=\left(r_{\|}, \mathbf{r}_{\perp}\right)$ with center at the origin, where $r_{\|}$and $\mathbf{r}_{\perp}$ are the components of $\mathbf{r}$ parallel and perpendicular to the symmetry axis. Using the notation $\left|\mathbf{r}_{\perp}\right|=r_{\perp}$, we introduce elliptic coordinates

$$
2 f \xi=r_{+}+r_{-}, \quad 2 f \eta=r_{+}-r_{-},
$$

where

$$
2 f=\sqrt{l^{2}-s^{2}}, \quad r_{ \pm}=\sqrt{\left(r_{\|} \pm f\right)^{2}+r_{\perp}^{2}},
$$

so that

$$
r_{\|}=f \xi \eta, \quad r_{\perp}=f \sqrt{\left(\xi^{2}-1\right)\left(1-\eta^{2}\right)} .
$$

Keeping $\xi>1$ constant defines in the $\left(r_{\|}, r_{\perp}>0\right)$ half plane a half ellipse with diameters $l_{\xi}=2 f \xi$ and $s_{\xi}=2 f \sqrt{\xi^{2}-1}$, where $\eta$ varies on the ellipse from $\eta=-1$ for $r_{\|}<0, r_{\perp}=0$ via $\eta=0$ for $r_{\|}=0$ to $\eta=1$ for $r_{\|}>0, r_{\perp}=0$. Thus the half plane corresponds to $1 \leqslant \xi \leqslant \infty,-1 \leqslant \eta \leqslant 1$. Points on the particle surface are determined by $\xi=\xi_{0}$, where

$$
\xi_{0}=1 / \sqrt{1-(s / l)^{2}},
$$

since $l_{\xi_{0}}=l, s_{\xi_{0}}=s$. The interior and exterior of the particle correspond to $1 \leqslant \xi<\xi_{0}$ and $\xi>\xi_{0}$, respectively.

When acting on the propagator in (3.6)

$$
g\left(\mathbf{r}, \mathbf{r}^{\prime}\right)=\widetilde{g}\left(r_{\|}, r_{\perp} ; r_{\|}^{\prime}, r_{\perp}^{\prime} ; c\right)=\hat{g}\left(\xi, \eta ; \xi^{\prime}, \eta^{\prime} ; c\right),
$$

where

$$
c \equiv \cos \theta=\mathbf{r}_{\perp} \mathbf{r}_{\perp}^{\prime} /\left(r_{\perp} r_{\perp}^{\prime}\right),
$$

the Laplacian reads

$$
\begin{aligned}
\Delta_{\mathbf{r}}= & \partial_{r_{\|}}^{2}+\partial_{r_{\perp}}^{2}+\frac{d-2}{r_{\perp}} \partial_{r_{\perp}}+\frac{1}{r_{\perp}^{2}} \mathcal{C} \\
= & \frac{1}{f^{2}\left(\xi^{2}-\eta^{2}\right)}\left\{\left[\left(\xi^{2}-1\right) \partial_{\xi}^{2}+(d-1) \xi \partial_{\xi}+\frac{1}{\xi^{2}-1} \mathcal{C}\right]\right. \\
& \left.-\left[\left(\eta^{2}-1\right) \partial_{\eta}^{2}+(d-1) \eta \partial_{\eta}+\frac{1}{\eta^{2}-1} \mathcal{C}\right]\right\},
\end{aligned}
$$

where

$$
\mathcal{C}=\left(1-c^{2}\right) \partial_{c}^{2}-(d-2) c \partial_{c} .
$$

When acting on the end density

$$
e(\mathbf{r})=\widetilde{e}\left(r_{\|}, r_{\perp}\right)=\hat{e}(\xi),
$$

only the $\xi$ derivatives in the Laplacian (3.13) contribute, and $\Delta e=0$ implies that $\partial_{\xi}\left[\left(\xi^{2}-1\right)^{(d-1) / 2} \partial_{\xi} \hat{e}\right]$ vanishes, so that

$$
\hat{e}(\xi)=1-\kappa(\xi) / \kappa\left(\xi_{0}\right),
$$

where

$$
\kappa(\xi)=\int_{\xi}^{\infty} \mathrm{d} \tau\left(\tau^{2}-1\right)^{-(d-1) / 2} .
$$

Expanding (3.16) for small particle size and comparing with the operator expansion (2.1) yield 


$$
\mathcal{I}=(2 f)^{d-2} \frac{1}{(d-2) \kappa\left(\xi_{0}\right)}
$$

and the relation

$$
\mathcal{N}^{\prime}=\frac{\Gamma(d / 2)}{(d-1) 2^{4-d} \pi^{d / 2}} \mathcal{N}+\frac{1}{2 d} f^{2} \mathcal{I},
$$

as we show in Appendix A. There we also evaluate the propagator $g$. Its small particle expansion is consistent with (3.18) and (3.19) and yields

$$
\mathcal{N}=\frac{\pi^{d / 2}}{d \Gamma(d / 2)} f^{d}\left\{\frac{1}{\lambda\left(\xi_{0}\right)}-\frac{1}{\kappa\left(\xi_{0}\right)}-\frac{1}{\mu\left(\xi_{0}\right)}\right\},
$$

where

$$
\begin{aligned}
& \lambda(\xi)=\int_{\xi}^{\infty} \mathrm{d} \tau \tau^{-2}\left(\tau^{2}-1\right)^{-(d-1) / 2}, \\
& \mu(\xi)=\int_{\xi}^{\infty} \mathrm{d} \tau\left(\tau^{2}-1\right)^{-(d+1) / 2} .
\end{aligned}
$$

For nearly spherical prolate ellipsoids, where $f \ll l$, $\xi_{0}$ $\gg 1$, the above expressions for $\mathcal{I}, \mathcal{N}^{\prime}$, and $\mathcal{N}$ are consistent with the results of first order in $(l-s) / l$ given in Eqs. (3.27), (3.29), and (3.30) of Ref. 10. In the needle limit where $f$ $\rightarrow l / 2$ and $\xi_{0} \rightarrow 1$, the above expressions yield the new results

$$
\mathcal{I} \rightarrow \frac{d-3}{d-2} l s^{d-3}
$$

and

$$
\mathcal{N}^{\prime} \rightarrow \frac{1}{8 d} \frac{d-3}{d-2} l^{3} s^{d-3}
$$

valid for $3<d$, and

$$
\mathcal{N} \rightarrow\left(\frac{\pi}{4}\right)^{(d-1) / 2} \frac{1}{d} \frac{(d-3)^{2}}{d-2} \Gamma\left(\frac{5-d}{2}\right) l^{6-d} s^{2(d-3)},
$$

for $3<d<5$. For more details see the end of Appendix A.

\section{B. Cylinder}

Here we calculate the universal amplitudes $J$ and $K$ of the cylinder for ideal polymers and show that the relations (2.9) and (2.10) between needle and cylinder amplitudes are indeed fulfilled.

The parallel Fourier transform $\mathcal{P}$ of the cylinder-induced propagator is given $b^{5}$

$$
\begin{aligned}
\mathcal{P}\left(p ; \mathbf{r}_{\perp}, \mathbf{r}_{\perp}^{\prime}\right) \equiv & \int_{-\infty}^{\infty} \mathrm{d} r_{\|} e^{i p\left(r_{\|}-r_{\|}^{\prime}\right)}\left[\langle\varphi\rangle_{\mathrm{cyl}}-\langle\varphi\rangle_{\mathrm{bulk}}\right] \\
= & -\sum_{\beta=0}^{\infty} \frac{1}{2 \pi^{(d-1) / 2}} \Gamma(\alpha) a C_{\beta}^{(\alpha)}(c) \\
& \times \frac{1}{\left(r_{\perp} r_{\perp}^{\prime}\right)^{\alpha}} K_{a}\left(|p| r_{\perp}\right) K_{a}\left(|p| r_{\perp}^{\prime}\right) \frac{I_{a}(|p| R)}{K_{a}(|p| R)}
\end{aligned}
$$

where $\beta$ is a non-negative integer, $\alpha=(d-3) / 2, a=\alpha+\beta$, the $C_{\beta}^{(\alpha)}$ are Gegenbauer polynomials, $c$ is from (3.12), and $I$ and $K$ are modified Bessel functions. The contributions of order $R^{2 \alpha}$ and $R^{4 \alpha}$ both arise from $\beta=0$ and must have the form

$$
\begin{aligned}
\left.\mathcal{P}\right|_{R^{2 \alpha}}+\left.\mathcal{P}\right|_{R^{4 \alpha}}= & \int_{-\infty}^{\infty} \mathrm{d} r_{\|} e^{i p\left(r_{\|}-r_{\|}^{\prime}\right)} \\
& \times\left\langle\varphi \cdot-R^{2 \alpha} J \int_{-\infty}^{\infty} \mathrm{d} r_{1 \|} \psi\left(r_{1 \|}, \mathbf{0}\right)-R^{4 \alpha} K\right. \\
& \left.\left.\times \int_{-\infty}^{\infty} \mathrm{d} r_{1 \|} \int_{-\infty}^{\infty} \mathrm{d} r_{2 \|} \psi\left(r_{1 \|}, \mathbf{0}\right) \psi\left(r_{2 \|}, \mathbf{0}\right)\right]\right\rangle_{\text {bulk }}
\end{aligned}
$$

because of (2.2). Using the Fourier transform of the bulk propagator,

$$
\begin{aligned}
\mathcal{P}_{\mathrm{b}}\left(p ; \mathbf{r}_{\perp}, \mathbf{r}_{\perp}^{\prime}\right) & \equiv \int_{-\infty}^{\infty} \mathrm{d} r_{\|} e^{i p\left(r_{\|}-r_{\|}^{\prime}\right)}\langle\varphi\rangle_{\text {bulk }} \\
& =\frac{1}{(2 \pi)^{(d-1) / 2}}\left(\frac{|p|}{\left|\mathbf{r}_{\perp}-\mathbf{r}_{\perp}^{\prime}\right|}\right)^{\alpha} K_{\alpha}\left(\left|p \| \mathbf{r}_{\perp}-\mathbf{r}_{\perp}^{\prime}\right|\right),
\end{aligned}
$$

and relating $\psi$ to $\boldsymbol{\Phi}^{2}$ as below (A18), one finds that (3.25) and (3.26) are consistent, provided

$$
J=\frac{1}{\sqrt{\pi}} 2^{d-2} \frac{\Gamma((d-2) / 2)}{\Gamma((d-3) / 2)}, \quad K=-\frac{1}{2} J^{2} .
$$

Here the factor $\mathcal{P}_{\mathrm{b}}(p ; \mathbf{0 , 0})$ in the Wick factorization of $\left.\mathcal{P}\right|_{R^{4 \alpha}}$ is obtained for $d>3$ by analytic continuation from $d<3$. We check in Appendix B that $\langle\psi\rangle_{\text {cyl }}$ is also reproduced by (2.2) and (3.28).

The Gaussian expressions (3.22)-(3.24) for the needle amplitudes $\mathcal{I}, \mathcal{N}$, and $\mathcal{N}^{\prime}$ and (3.28) for the cylinder amplitudes $J$ and $K$ are consistent with the general needle-cylinder relations (2.9) and (2.10). To check (2.10) one uses that ${ }^{18}$

$$
\frac{C_{\psi \psi T T}}{B_{T}}=-2^{5-2 d} \pi^{d / 2} \frac{1}{\Gamma((d-2) / 2)}
$$

and

$$
\begin{aligned}
& \int_{-1}^{1} \mathrm{~d} \varrho_{1} \int_{-1}^{1} \mathrm{~d} \varrho_{2}\left[\left(1-\varrho_{1}^{2}\right)\left(1-\varrho_{2}^{2}\right)\right]^{(d-3) / 2}\left|\varrho_{1}-\varrho_{2}\right|^{4-d} \\
& \quad=\frac{4 \sqrt{\pi}}{d \Gamma(d / 2)}\left[\Gamma\left(\frac{d-1}{2}\right)\right]^{2} \Gamma\left(\frac{5-d}{2}\right)
\end{aligned}
$$

\section{Stress tensor weight for the needle in $d>5$}

For a needle interacting with ideal polymers in $d>5$, Eq. (3.20) and the observations at the end of Appendix A yield

$$
\mathcal{N} \rightarrow 4\left(\frac{\pi}{4}\right)^{d / 2} \frac{1}{d \Gamma(d / 2)} \frac{1}{d-5} l s^{d-1}
$$

for the stress tensor weight, which should be compared with the result (3.24) for $3<d<5$. While $\mathcal{N}$ is positive in both 
cases, the $d$ dependence of the amplitudes and the power laws in $l$ and $s$ are different.

Again this can be understood in terms of cylinder properties. In addition to the second and higher powers of $\int \mathrm{d} r_{\|} \psi$ there is also a term ${ }^{19}$

$$
-\mathcal{S} \int_{-\infty}^{\infty} \mathrm{d} r_{\|} T_{\|\|}\left(r_{\|}, \mathbf{0}\right)
$$

on the right hand side of the cylinder expansion (2.2). Since $\mathcal{S} \rightarrow S R^{d-1}$, with a universal amplitude $S$, it contributes

$$
S\left(\frac{s}{2}\right)^{d-1} \frac{l}{2} \sqrt{\pi} \frac{\Gamma((d+1) / 2)}{\Gamma((d+2) / 2)}
$$

to the coefficient $\mathcal{N}$ in the needle expansion (2.1). Here the factor following $l / 2$ equals $\int_{-1}^{1} \mathrm{~d} \varrho[\sigma(\varrho)]^{d-1}$ with $\sigma$ from (2.6).

The contribution (3.33) to $\mathcal{N}$ arising from $\int \mathrm{d} r_{\|} T_{\|\| \|}$should be compared with the corresponding contribution (2.10) arising from $\left(\int \mathrm{d} r_{\|} \psi\right)^{2}$. For the Gaussian model of ideal polymers and a thin needle with $s \rightarrow 0$, the former and latter dominate for $d>5$ and $d<5$, since $d-1<2\left(x_{\epsilon}-1\right)$ and $2\left(x_{\epsilon}-1\right)<d$ -1 , respectively. In Appendix B we calculate the amplitude $S$ and show that (3.33) implies (3.31).

\section{SELF-AVOIDING POLYMERS AND EFFECTIVE NEEDLE-WALL INTERACTION}

The polymer-induced effective interaction between an ellipsoid and a wall has been discussed in Ref. 10. Here we concentrate on a dilute polymer solution and on distances $z$ between particle and wall that are much smaller than the polymer size $\mathcal{R}_{x}$. In the corresponding parameter range

$$
s \ll l \ll z \ll \mathcal{R}_{x}
$$

for a small needle, the leading free energy of interaction

$$
\delta F_{\text {iso }}=p_{0} \frac{A}{2^{m}}\left(-\mathcal{R}_{x}^{1 / \nu}+B z^{1 / \nu}\right) \mathcal{I},
$$

with $\mathcal{I} \rightarrow I l s^{m-1}$, is isotropic, while the orientation-dependent interaction is the sum of the two power law contributions ${ }^{20}$

$$
\delta F_{\text {ori }}=p_{0}(\cos \vartheta)^{2} \frac{d}{d-1} \mathcal{N}
$$

and

$$
\delta F_{\text {ori }}^{\prime}=p_{0}(\cos \vartheta)^{2} \frac{A}{2^{m}} B \frac{1}{\nu}\left(\frac{1}{\nu}-1\right) z^{(1 / \nu)-2} \mathcal{N}^{\prime}
$$

arising from the $\mathcal{N}$ and $\mathcal{N}^{\prime}$ terms in Eq. (2.1) with $\mathcal{N}$ $\rightarrow N l^{d}(s / l)^{2(m-1)}$ and $\mathcal{N}^{\prime} \rightarrow N^{\prime} l^{3} s^{m-1}$. Here $I, N$, and $N^{\prime}$ are needle amplitudes determined by Eqs. (2.9) and (2.10) with $x_{\epsilon} \rightarrow m, \vartheta$ is the angle between the axis of the needle and the surface normal of the wall, and $A$ and $B$ are the amplitudes ${ }^{3}$ in the free energy $p_{0} A R_{\text {sphere }}^{m} \mathcal{R}_{x}^{1 / \nu}$ it costs to insert a sphere ${ }^{21}$ with small radius $R_{\text {sphere }}$ in the unbounded polymer solution and in the asymptotic form $B\left(z / \mathcal{R}_{x}\right)^{1 / \nu}$ of the bulknormalized polymer-density profile close to the planar boundary wall of the half space without a particle. The universal amplitudes $A, B, I, N$, and $N^{\prime}$ and the factor $(1 / \nu)-1$ are all positive. Thus $\delta F_{\text {ori }}$ and $\delta F_{\text {ori }}^{\prime}$ both tend to align the particle parallel to the wall.

For ideal chains with $1 / \nu=2$ and $m=d-2$, the $z$ dependence in (4.4) drops out and $\mathcal{N} / \mathcal{N}^{\prime}$ is small for $s / l$ sufficiently ${ }^{22}$ small. Then $\delta F_{\text {ori }}^{\prime}$ dominates in the whole range (4.1).

Things are more involved for self-avoiding chains in $d$ $=3$, where the ratio $\delta F_{\text {ori }} / \delta F_{\text {ori }}^{\prime}$ is proportional to $\left(s z / l^{2}\right)^{0.30}$ since $m-1=2-(1 / \nu)=0.30$. Thus in the range (4.1) both power laws $\delta F_{\text {ori }}$ and $\delta F_{\text {ori }}^{\prime}$ contribute to the orientational behavior, in general. In the subranges with $l / z \ll s / l$ and $s / l \ll l / z$, the dominant orientational behavior comes from $\delta F_{\text {ori }}$ and $\delta F_{\text {ori }}^{\prime}$, respectively.

\section{SUMMARY AND CONCLUDING REMARKS}

We have studied the interaction between long flexible polymers and nonspherical colloidal particles in the form of ellipsoids, cylinders, and needles. We consider the so-called protein or nanoparticle limit where the sizes of ellipsoids and needles and the diameter of the cylinder are mesoscopic but small compared to the polymer size $\mathcal{R}_{x}$ and where the interaction can be described by field theoretic operator expansions $^{3,5}$ such as (2.1) and (2.2) in Sec. II. These are similar to multipole expansions in electrostatics and involve polymer-colloid interaction strengths that depend on the particle size and shape. We augment previous studies ${ }^{3,5,10}$ of anisotropic particles by considering needles and cylinders interacting with polymers in a good solvent.

In particular, we show that the orientation-dependent effective interaction induced by polymers between a thin needle and a wall is determined by the interaction of the polymers with a single cylinder. This follows from the exact relations (2.9) and (2.10) between the polymer-colloid interaction strengths for needles and cylinders that are derived by comparing the corresponding operator expansions. We use the cylinder expansion in (2.2) and (3.32), which is extended beyond the leading order of Ref. 5 , and interpret it in a local way. For the needle of width $s$ and length $l$ with $s \ll l$, the relations allow us to predict not only the power law exponents in $s$ and $l$ of the leading isotropic and anisotropic interactions but also their universal amplitudes from properties of the cylinder.

There are two anisotropic polymer-colloid interactions, with strengths $\mathcal{N}$ and $\mathcal{N}^{\prime}$, in (2.1). Both are important if the colloid is a thin needle. To illustrate this we consider in Sec. IV needle-wall distances $z$ large compared to $l$ but small compared to the polymer size $\mathcal{R}_{x}$, for which the orientationdependent part of the effective particle-wall interaction free energy $\delta F$ is independent ${ }^{10}$ of $\mathcal{R}_{x}$, and show that for selfavoiding polymers in $d=3$ it is a sum of the two power laws $\propto s^{0.60} l^{2.40} z^{0}$ and $\propto s^{0.30} l^{3} z^{-0.30}$ which arise from $\mathcal{N}$ and $\mathcal{N}^{\prime}$ and are given in Eqs. (4.3) and (4.4) with their universal amplitudes included. The first and second power laws both orient the needle parallel to the wall and dominate in the subranges of (4.1) with $l / z \ll s / l$ and $s / l \ll l / z$, respectively.

The above result for the needle should be compared with 
the corresponding result ${ }^{10}$ for a thin circular disk of diameter $l$ which is also oriented parallel to the wall. The latter is independent of the width of the disk and given by the single $e^{23}$ term $\propto l^{3} z^{0}$ in the entire range $l \ll z \ll \mathcal{R}_{x}$.

To confirm the reasoning leading to the fundamental relations (2.9) and (2.10), we check them in Sec. III for the case of ideal chains, i.e., for the Gaussian model. Here we consider $d>3$, since both self-avoiding chains in $d=3$ and ideal chains in $d>3$ show power law behavior ${ }^{6}$ in the cylinder radius $R$.

The peculiar change of the stress tensor amplitude $\mathcal{N}$ in (3.20) for the thin needle from the form (3.24) for $3<d$ $<5$ to (3.31) for $d>5$ can be traced back to the cylinder operator expansion (2.2). The $\mathcal{K}$ and $\mathcal{S}$ terms in (2.2) and (3.32) both contribute to $T_{\|\|}$in the needle expansion (2.1), but there is a crossing of the $R$ exponents $2(m-1)=2(d-3)$ and $d-1$ of $\mathcal{K}$ and $\mathcal{S}$ at $d=5$.

The new ideal chain expressions in Sec. III and Appendix A provide a starting point for perturbative expansions about the upper critical dimension $d=4$ of polymer-ellipsoid interaction strengths for self-avoiding chains. The corresponding expansions for the cylinder strength $J$ in (2.2) and the expressions in (2.9) for the needle strengths $\mathcal{I}$ and $\mathcal{N}^{\prime}$ are determined by the $\varepsilon$ expansion of the related ${ }^{15}$ universal amplitudes $A_{d_{\perp}, d}$ given in Eq. (B8) of the second article in Ref. 5. It would be interesting to generalize the method in Appendix $B$ of the above article in order to check the power law $R^{2\left(x_{\epsilon}-1\right)}$ in (2.2) and to calculate the $\varepsilon$ expansion of $K$, which enters the expression (2.10) for the needle strength $\mathcal{N}$.

The known ${ }^{3}$ logarithmic behavior of the needle amplitudes for ideal polymers in $d=3$ is also contained in the expressions (3.18)-(3.20), as shown near the end of Appen$\operatorname{dix}$ A.

\section{ACKNOWLEDGMENT}

It is my pleasure to thank T. W. Burkhardt for his interest and useful discussions.

\section{APPENDIX A: PROPAGATOR FOR AN ELLIPSOIDAL PARTICLE}

The Gaussian propagator outside a prolate ellipsoid in $d$ spatial dimensions and with Dirichlet boundary conditions follows from Eqs. (3.3), (3.6), (3.11), (3.13), and (3.14) by means of standard arguments ${ }^{24}$ and is given by

$$
\begin{aligned}
\hat{g}\left(\xi, \eta ; \xi^{\prime}, \eta^{\prime} ; c\right)= & f^{2-d} \sum_{\beta, \gamma=0}^{\infty} \mathcal{A}_{\beta, \gamma} C_{\beta}^{(\alpha)}(c) S_{\beta, \gamma}(\eta) S_{\beta, \gamma}\left(\eta^{\prime}\right) \\
& \times\left\{R_{\beta, \gamma}^{(1)}\left(\xi_{\min }\right) R_{\beta, \gamma}^{(\infty)}\left(\xi_{\max }\right)\right. \\
& \left.-\frac{R_{\beta, \gamma}^{(1)}\left(\xi_{0}\right)}{R_{\beta, \gamma}^{(\infty)}\left(\xi_{0}\right)} R_{\beta, \gamma}^{(\infty)}(\xi) R_{\beta, \gamma}^{(\infty)}\left(\xi^{\prime}\right)\right\} .
\end{aligned}
$$

Here $\beta$ and $\gamma$ are independent non-negative integers, $\alpha$ equals $(d-3) / 2$, the $C_{\beta}^{(\alpha)}$ are Gegenbauer polynomials as in Eq. (3.25), $\xi_{\min }$ and $\xi_{\max }$ denote the smaller and larger of $\xi$ and $\xi^{\prime}$, and

$$
\begin{aligned}
& S_{\beta, \gamma}(\eta)=\left(1-\eta^{2}\right)^{\beta / 2} C_{\gamma}^{(b)}(\eta), \\
& R_{\beta, \gamma}^{(1)}(\xi)=\left(\xi^{2}-1\right)^{\beta / 2} C_{\gamma}^{(b)}(\xi),
\end{aligned}
$$

where

$$
b=\beta+(d / 2)-1 .
$$

Both $R^{(1)}$ and $R^{(\infty)}$ satisfy the differential equation ${ }^{25}$

$$
\begin{aligned}
& {\left[\left(\xi^{2}-1\right) \partial_{\xi}^{2}+(d-1) \xi \partial_{\xi}-\frac{\beta(\beta+d-3)}{\xi^{2}-1}\right.} \\
& \quad-(\beta+\gamma)(\beta+\gamma+d-2)] R_{\beta, \gamma}(\xi)=0 .
\end{aligned}
$$

While the solution $R^{(1)}$ remains finite for $\xi \backslash 1$, the solution $R^{(\infty)}$ decays for large $\xi$ and diverges ${ }^{26}$ for $\xi \searrow 1$ as

$$
R_{\beta, \gamma}^{(\infty)}(\xi) \propto\left[\xi^{(\beta+\gamma+d-2)},(\xi-1)^{-(\beta+d-3) / 2}\right], \quad \xi \rightarrow[\infty, 1] .
$$

For later use we note the special cases

$$
R_{0,0}^{(1)}(\xi)=1, \quad R_{1,0}^{(1)}(\xi)=\sqrt{\xi^{2}-1}, \quad R_{0,1}^{(1)}(\xi)=(d-2) \xi,
$$

and

$$
\begin{aligned}
& R_{0,0}^{(\infty)}(\xi)=\kappa(\xi), \\
& R_{1,0}^{(\infty)}(\xi)=\sqrt{\xi^{2}-1} \mu(\xi), \quad R_{0,1}^{(\infty)}(\xi)=\frac{\xi}{d-2} \lambda(\xi),
\end{aligned}
$$

where $\kappa, \mu$, and $\lambda$ are the integrals defined in Eqs. (3.17) and (3.21). For convenience the constant prefactors in $R^{(\infty)}$ have been chosen so that $w_{0,0}=w_{1,0}=w_{0,1}=1$. Here

$$
\begin{aligned}
w_{\beta, \gamma} & =\left(\xi^{2}-1\right)^{(d-1) / 2}\left\{\left[\partial_{\xi} R_{\beta, \gamma}^{(1)}(\xi)\right] R_{\beta, \gamma}^{(\infty)}(\xi)\right. \\
& \left.-R_{\beta, \gamma}^{(1)}(\xi)\left[\partial_{\xi} R_{\beta, \gamma}^{(\infty)}(\xi)\right]\right\}
\end{aligned}
$$

is independent of $\xi$ and related to the Wronskian of (A4). Finally, the amplitudes in (A1) are

$$
\mathcal{A}_{\beta, \gamma}=C_{\beta}^{(\alpha)}(1) /\left[\Omega_{d-2} h_{\beta}^{(\alpha)} h_{\gamma}^{(b)} w_{\beta, \gamma}\right],
$$

where $\Omega_{D}=2 \pi^{D / 2} / \Gamma(D / 2)$ is the surface area of the sphere in $D$ spatial dimensions with radius 1 , and the

$$
h_{j}^{(v)}=\frac{2 \pi}{j !} \frac{2^{-2 v} \Gamma(j+2 v)}{(j+v)[\Gamma(v)]^{2}}
$$

are coefficients appearing in the orthogonality relations

$$
\int_{-1}^{1} \mathrm{~d} c\left(1-c^{2}\right)^{\alpha-(1 / 2)} C_{\beta}^{(\alpha)}(c) C_{\beta^{\prime}}^{(\alpha)}(c)=\delta_{\beta, \beta^{\prime}} h_{\beta}^{(\alpha)}
$$

and

$$
\int_{-1}^{1} \mathrm{~d} \eta\left(1-\eta^{2}\right)^{\alpha} S_{\beta, \gamma}(\eta) S_{\beta, \gamma^{\prime}}(\eta)=\delta_{\gamma, \gamma^{\prime}} h_{\gamma}^{(b)} .
$$

The first and second terms in the braces of (A1) correspond to the bulk and ellipsoid-induced parts, respectively, of the propagator $g=g_{\text {bulk }}+g_{\text {ind }}$. 
We now evaluate the amplitudes $\mathcal{I}, \mathcal{N}$, and $\mathcal{N}^{\prime}$ by expanding $g\left(\mathbf{r}, \mathbf{r}^{\prime}\right)$ and $e(\mathbf{r})$ for particle sizes much smaller ${ }^{27}$ than $r$ and $r^{\prime}$. In this case $f \ll r, r^{\prime}$, and

$$
\xi \rightarrow \frac{r}{f}\left(1+\frac{f^{2} r_{\perp}^{2}}{2 r^{4}}+\cdots\right)
$$

becomes large, while

$$
\eta \rightarrow \frac{r_{\|}}{r}(1+\cdots)
$$

is independent of $f$ in leading order.

The contributions to $g_{\text {ind }}$ up to order $f^{d}$ are contained in the terms $(\beta, \gamma)=(0,0),(1,0)$, and $(0,1)$, see (A5) and (A13), and are given by

$$
\begin{aligned}
g_{\text {ind }(0,0)} \rightarrow & -\frac{\tilde{S}_{d}}{\kappa\left(\xi_{0}\right)} \frac{f^{d-2}}{\left(r r^{\prime}\right)^{d-2}} \\
& \times\left\{\frac{1}{d-2}+\frac{f^{2}}{2}\left[-\frac{1}{d}\left(\frac{1}{r^{2}}+\frac{1}{r^{\prime 2}}\right)+U\right]\right\}
\end{aligned}
$$

and

$$
\begin{aligned}
\left\{\left.g_{\text {ind }}\right|_{(1,0)},\left.g_{\text {ind }}\right|_{(0,1)}\right\} \rightarrow & -\frac{f^{d}}{\left(r r^{\prime}\right)^{d-1}} \widetilde{S}_{d} \frac{d-2}{d} \\
& \times\left\{\frac{1}{\mu\left(\xi_{0}\right)}\left(\frac{\mathbf{r r}^{\prime}}{r r^{\prime}}-V\right), \frac{1}{\lambda\left(\xi_{0}\right)} V\right\},
\end{aligned}
$$

with the amplitude $\widetilde{S}_{d}=\Gamma((d-2) / 2) /\left(4 \pi^{d / 2}\right)$ in the Gaussian bulk propagator $\langle\varphi\rangle_{\text {bulk }}=\widetilde{S}_{d}\left|\mathbf{r}-\mathbf{r}^{\prime}\right|^{2-d}$ and the spatially anisotropic expressions

$$
U=\frac{r_{\|}^{2}}{r^{4}}+\frac{r_{\|}^{\prime 2}}{r^{\prime 4}}, \quad V=\frac{r_{\|} r_{\|}^{\prime}}{r r^{\prime}} .
$$

The bracket on the right hand side of (A16) equals $\mathbf{r}_{\perp} \mathbf{r}_{\perp}^{\prime} /\left(r r^{\prime}\right)$ and comes from $c \sqrt{1-\eta^{2}} \sqrt{1-\eta^{\prime 2}}$ in $\left.g_{\text {ind }}\right|_{(1,0)}$ on using (3.12) and (A14).

Identifying the contribution in (A15) of order $f^{d-2}$ with

$$
-\mathcal{I}\langle\varphi \psi(0)\rangle_{\mathrm{bulk}}=-\mathcal{I} 2^{2-d} \widetilde{S}_{d}\left(r r^{\prime}\right)^{2-d}
$$

leads to the result (3.18). Here we have used the operator expansion (2.1) and the definition of $\psi$ below (2.2), with $\mathcal{A}_{\epsilon}=2^{(3 / 2)-d}$ and $-\epsilon / B_{\epsilon}^{1 / 2}=\Phi^{2} /\left(\sqrt{2} \tilde{S}_{d}\right)$ in the one-component Gaussian model, see Sec. III B of Ref. 17.

The sum of the anisotropic $U$ and $V$ terms in (A15) and (A16) is to be identified with the anisotropic contribution in

$$
-\mathcal{N}\left\langle\varphi T_{\|\|}(0)\right\rangle_{\text {bulk }}-\mathcal{N}^{\prime}\left\langle\varphi \partial_{\|}^{2} \psi(0)\right\rangle_{\text {bulk }},
$$

where the bulk three-point functions are given, e.g., in Sec. III B and Appendixes A and C of ${ }^{18}$ Ref. 17. Substituting in (A15) and (A16) the relations

$$
U=\frac{1}{2(d-1)}[\omega-\tau]+\text { iso }
$$

$$
V=\frac{r r^{\prime}}{4(d-1)(d-2)}[d \tau+(d-2) \omega]+\text { iso, }
$$

where "iso" denotes spatially isotropic contributions and

$$
\begin{aligned}
& \tau=\left\langle\varphi T_{\|\|}(0)\right\rangle_{\text {bulk }} \frac{2}{\tilde{S}_{d}^{2}} \frac{(d-1)}{d(d-2)}\left(r r^{\prime}\right)^{d-2}, \\
& \omega=\left\langle\varphi \partial_{\|}^{2} \psi(0)\right\rangle_{\text {bulk }} \frac{2^{d-2}}{(d-2) \tilde{S}_{d}}\left(r r^{\prime}\right)^{d-2},
\end{aligned}
$$

and comparing with (A19) lead to the results (3.19) and (3.20).

It is instructive to confirm that the leading isotropic and anisotropic behavior

$$
\left[(e(\mathbf{r})-1)_{\text {iso }}, e(\mathbf{r})_{\text {aniso }}\right]=-\frac{1}{\kappa\left(\xi_{0}\right)}\left[\frac{1}{d-2}\left(\frac{f}{r}\right)^{d-2}, \frac{r_{\|}^{2}}{2 r^{2}}\left(\frac{f}{r}\right)^{d}\right]
$$

of the end density $e(\mathbf{r})$ of ideal chains in (3.15) and (3.16) is also reproduced by the operator expansion (2.1) with the amplitudes $\mathcal{I}, \mathcal{N}$, and $\mathcal{N}^{\prime}$ obtained above. This is equivalent to confirming that

$$
\begin{aligned}
& {\left[(e(\mathbf{r})-1)_{\text {iso }}, e(\mathbf{r})_{\text {aniso }}\right]} \\
& =-\lim _{L \rightarrow \infty} \mathcal{L}_{t \rightarrow L} \int \mathrm{d} \mathbf{r}^{\prime}\left[\left\langle\varphi \beta_{\mathrm{I}} O_{\mathrm{I}}\right\rangle_{\text {bulk }},\right. \\
& \left.\quad\left\langle\varphi \beta_{\mathrm{VII}} O_{\mathrm{VII}}\right\rangle_{\text {bulk,aniso }}\right]_{t>0},
\end{aligned}
$$

where $\mathcal{L}_{t \rightarrow L}$ is the inverse of the Laplace transform in (3.1). Here the operator terms in (2.1),

$$
\left[\mathcal{I} \psi, \mathcal{N} T_{\|\|}+\mathcal{N}^{\prime} \partial_{\|}^{2} \psi\right]=\left[\beta_{\mathrm{I}} O_{\mathrm{I}}, \beta_{\mathrm{VI}} O_{\mathrm{VI}}+\beta_{\mathrm{VII}} O_{\mathrm{VII}}+\text { iso }\right],
$$

have been expressed in terms of the operators

$$
\left[O_{\mathrm{I}}, O_{\mathrm{VI}}, O_{\mathrm{VII}}\right]=\left[\Phi^{2},\left(\partial_{\|} \Phi\right)^{2}, \Phi\left(\partial_{\|}^{2} \Phi\right)\right] / 2
$$

introduced in Ref. 3 and the corresponding amplitudes

$$
\beta_{\mathrm{I}}=\mathcal{I} 2^{2-d} / \tilde{S}_{d}
$$

and

$$
\begin{aligned}
& \beta_{\mathrm{VI}}=\mathcal{N} d /(d-1)+\mathcal{N}^{\prime} 2^{3-d} / \widetilde{S}_{d}, \\
& \beta_{\mathrm{VII}}=-\mathcal{N}(d-2) /(d-1)+\mathcal{N}^{\prime} 2^{3-d} / \widetilde{S}_{d} .
\end{aligned}
$$

This is convenient since $O_{\mathrm{VI}}$ does not contribute to the integral over $\mathbf{r}^{\prime}$ in (A23).

With Wick's theorem and

$$
\int \mathrm{d} \mathbf{r}^{\prime}\left\langle\Phi\left(\mathbf{r}^{\prime}\right) \Phi\right\rangle_{\text {bulkt }>0}=1 / t,
$$

the right hand side of (A23) becomes 


$$
-\left[\beta_{\mathrm{I}}\langle\Phi(\mathbf{r}) \Phi\rangle_{\text {bulk }},\left(\beta_{\mathrm{VII}} / 2\right)\left\langle\Phi(\mathbf{r}) \partial_{\|}^{2} \Phi\right\rangle_{\text {bulk,aniso }}\right]_{t=0},
$$

since for large $L$ only $t=0$ survives. Substituting $\left[\langle\Phi(\mathbf{r}) \Phi\rangle_{\text {bulk }}\right]_{t=0}=\widetilde{S}_{d} / r^{d-2}$ in (A29) confirms Eq. (A23) provided that

$$
\beta_{\mathrm{VII}}=f^{2} \beta_{\mathrm{I}} / d
$$

which is in agreement with (3.19).

The amplitudes $\mathcal{I}, \mathcal{N}^{\prime}$, and $\mathcal{N}$ for a needle follow from Eqs. (3.18)-(3.20) as $\xi_{0}-1 \rightarrow(s / l)^{2} / 2$ becomes small. In (3.20) the leading contributions of $1 / \lambda$ and $1 / \kappa$ cancel in the difference, and it is convenient to write it as $1 / \lambda-1 / \kappa$ $=\delta /[\kappa(\kappa-\delta)]$ with $\delta=\kappa-\lambda . \quad$ For $2<d<5, \quad \delta \rightarrow \Gamma((5$ $-d) / 2) \Gamma((d-2) / 2) / \sqrt{\pi}$ tends to a constant, while $\delta$ $\rightarrow(s / l)^{-(d-5)} /(d-5)$ for $d>5$. The term $-1 / \mu \rightarrow-(s / l)^{d-1}(d$ -1 ) in (3.20) can be neglected for $2<d<5$ but is of the same order as $1 / \lambda-1 / \kappa$ for $d>5$. For $d>3$, where $\kappa$ $\rightarrow(s / l)^{-(d-3)} /(d-3)$, this leads to the results (3.24) and (3.31) for $\mathcal{N}$.

For $d=3$, where $\delta=1$ and $\kappa \rightarrow \ell$, with $\ell=\ln (2 l / s)$, one obtains $1 / \lambda-1 / \kappa \rightarrow 1 /[\ell(\ell-1)]$ and $\mathcal{N} \rightarrow l^{3} \pi /[12 \ell(\ell-1)]$. This and the corresponding results $\mathcal{I} \rightarrow l / \ell$ and $\mathcal{N}^{\prime} \rightarrow l^{3}\{1$ $+1 /[4(\ell-1)]\} /(24 \ell)$, which follow from (3.18) and (3.19), are consistent with (A27) and (A30) and the results $\beta_{\mathrm{VI}}$ $\rightarrow l^{3} \pi /[6(\ell-1)]$ and $\beta_{\mathrm{VII}} \rightarrow l^{3} \pi /(6 \ell)$ in the first article in Ref. 8. Note that $\left(\beta_{\mathrm{VI}} / \beta_{\mathrm{VII}}\right)-1 \rightarrow 1 /(\ell-1)$ reduces to the expression given in Eq. (195) of Ref. 3 only for $\ell \gg 1$.

\section{APPENDIX B: STRESS TENSOR AND ENERGY DENSITIES OUTSIDE A CYLINDER}

Here we evaluate the amplitude $\mathcal{S}$ in the term (3.32) of the operator-integral expansion (2.2) for the cylinder by cal- culating the contribution of order $R^{d-1}$ to the stress tensor density profile $\left\langle T_{k l}\right\rangle_{\mathrm{cyl}}$ outside the cylinder and comparing with the corresponding prediction

$$
\begin{aligned}
\left.\left\langle T_{k l}(\mathbf{r})\right\rangle_{\mathrm{cyl}}\right|_{\propto R^{d-1}}= & -S R^{d-1} \int_{-\infty}^{\infty} \mathrm{d} r_{1 \|} \\
& \times\left\langle T_{k l}\left(r_{\|}, \mathbf{r}_{\perp}\right) T_{\|\|}\left(r_{1 \|}, \mathbf{0}\right)\right\rangle_{\text {bulk }}
\end{aligned}
$$

of (3.32). Apart from (B1) we also expect from the expansion (2.2) a contribution of order $R^{2\left(x_{\epsilon}-1\right)}$ to $\left\langle T_{k l}\right\rangle_{\mathrm{cyl}}$ but none ${ }^{28}$ of order $R^{x_{\epsilon}-1}$. The contribution of order $R^{y}$ to $\left\langle T_{k l}\right\rangle_{\mathrm{cyl}}$ has the form

$$
\left.\left\langle T_{k l}(\mathbf{r})\right\rangle_{\mathrm{cyl}}\right|_{\propto R^{y}}=a_{y} \frac{R^{y}}{r_{\perp}^{d+y}} M_{k l}^{(y)}\left(r_{\perp}\right),
$$

where

$$
M_{k l}^{(y)}\left(r_{\perp}\right)=(y+2) \delta_{k l}-(d y+d-y) \delta_{k\|\|} \delta_{l \|}-(y+d) \frac{r_{\perp k} r_{\perp l}}{r_{\perp}^{2}} .
$$

This follows from the translation and rotation symmetries of the cylinder and from the scaling dimension $d$, the vanishing trace, and the continuity equation of the stress tensor. For $y$ $=d-1$,

$$
a_{d-1}=S B_{T} \frac{\sqrt{\pi} \Gamma(d-(1 / 2))}{\Gamma(d+2)}
$$

is related to $S$ in Eq. (B1) as follows from the known form of the bulk two-point function of $T$ which contains a universal amplitude $B_{T}$, see, e.g., Eq. (2.17) in Ref. 17.

For the Gaussian model the left hand side of (B1) can be calculated directly from the contribution

$$
\begin{aligned}
\left.\left.P\left(r_{\|}-r_{\|}^{\prime} ; \mathbf{r}_{\perp}, \mathbf{r}_{\perp}^{\prime}\right)\right|_{\propto R^{d-1}} \equiv\langle\varphi\rangle_{\mathrm{cyy}}\right|_{\propto R^{d-1}}= & -\frac{1}{2 \pi^{(d-1) / 2}} \frac{1}{\left(r_{\perp} r_{\perp}^{\prime}\right)^{\alpha}} \int_{0}^{\infty} \frac{\mathrm{d} p}{\pi} \cos \left[p\left(r_{\|}-r_{\|}^{\prime}\right)\right] \\
& \times\left(\frac{p R}{2}\right)^{d-1} \frac{4}{\Gamma(\alpha+1)}\left[\frac{\alpha^{2}}{\alpha^{2}-1} K_{\alpha}\left(p r_{\perp}\right) K_{\alpha}\left(p r_{\perp}^{\prime}\right)+\frac{\mathbf{r}_{\perp} \mathbf{r}_{\perp}^{\prime}}{r_{\perp} r_{\perp}^{\prime}} K_{\alpha+1}\left(p r_{\perp}\right) K_{\alpha+1}\left(p r_{\perp}^{\prime}\right)\right]
\end{aligned}
$$

of order $R^{d-1}$ of the propagator in (3.25) and the explicit form of the stress tensor with ${ }^{29}$ canonical and improvement term.

A convenient way to calculate $a_{d-1}$ and $S$ is via the combination of matrix elements,

$$
\begin{aligned}
\left\langle T_{\|\|}\left(r_{\|}, \mathbf{r}_{\perp}\right)-T_{\text {per per }}\left(r_{\|}, \mathbf{r}_{\perp}\right)\right\rangle_{\mathrm{cyl}} & \\
= & \left.\partial_{r_{\|}} \partial_{r_{\|}^{\prime}} P\left(r_{\|}-r_{\|}^{\prime} ; \mathbf{r}_{\perp}, \mathbf{r}_{\perp}\right)\right|_{r_{\|}^{\prime} \rightarrow r_{\|}} \\
& -\left.\partial_{r_{\mathrm{per}}} \partial_{r_{\mathrm{per}}^{\prime}} P\left(0 ; \widetilde{\mathbf{r}}_{\perp}, \widetilde{\mathbf{r}}_{\perp}^{\prime}\right)\right|_{r_{\mathrm{per}}, r_{\mathrm{per}}^{\prime} \rightarrow 0} \\
& +\left.\frac{d-2}{4(d-1)} \partial_{r_{\mathrm{per}}}^{2} P\left(0 ; \widetilde{\mathbf{r}}_{\perp}, \widetilde{\mathbf{r}}_{\perp}\right)\right|_{r_{\mathrm{per}} \rightarrow 0} .
\end{aligned}
$$

Here "per" denotes a Cartesian direction along the unit vector $\mathbf{e}_{\text {per }}$ perpendicular to $\mathbf{r}_{\perp}$ and the cylinder axis $\|, \widetilde{\mathbf{r}}_{\perp}=\mathbf{r}_{\perp}$ $+\mathbf{e}_{\mathrm{per}} r_{\mathrm{per}}$, and $\widetilde{\mathbf{r}}_{\perp}^{\prime}=\mathbf{r}_{\perp}+\mathbf{e}_{\mathrm{per}} r_{\mathrm{per}}^{\prime}$. The twofold derivative in the last term equals $r_{\perp}^{-1}\left(\mathrm{~d} / \mathrm{d} r_{\perp}\right) P\left(0 ; \mathbf{r}_{\perp}, \mathbf{r}_{\perp}\right)$. For the contribution of order $R^{d-1}, P$ in (B6) is given by the expression in (B5). In the mixed derivative $\partial_{r_{\mathrm{per}}} \partial_{r_{\mathrm{per}}^{\prime}} P$ only the term

$$
\left(\partial_{r_{\mathrm{per}}} \partial_{r_{\mathrm{per}}^{\prime}} \frac{\widetilde{\mathbf{r}}_{\perp} \widetilde{\mathbf{r}}_{\perp}^{\prime}}{\widetilde{r}_{\perp} \widetilde{r}_{\perp}^{\prime}}\right)_{r_{\mathrm{per}}, r_{\mathrm{per}}^{\prime} \rightarrow 0}=\frac{1}{r_{\perp}^{2}}
$$

survives, since $\partial_{r_{\text {per }}} \widetilde{r}_{\perp}$ vanishes for $r_{\text {per }} \rightarrow 0$. After the evaluation of the $p$ integrals, ${ }^{30}$ the three terms on the right hand side of (B6) are given by 


$$
\begin{gathered}
a_{d-1}\left(R^{d-1} / r_{\perp}^{2 d-1}\right)\left\{-8 \alpha^{5}-36 \alpha^{4}-44 \alpha^{3}+6 \alpha^{2}\right. \\
+37 \alpha+15,8 \alpha^{4}+24 \alpha^{3}+8 \alpha^{2}-24 \alpha-16, \\
\left.8 \alpha^{5}+28 \alpha^{4}+20 \alpha^{3}-18 \alpha^{2}-23 \alpha-6\right\},
\end{gathered}
$$

where

$$
a_{d-1}=\frac{[\Gamma(d / 2)]^{2} \Gamma(d-(1 / 2))}{2 \pi^{d / 2} \Gamma((d+1) / 2) \Gamma(d)(d+1)(d-1)(d-5)} .
$$

The sum of the terms in the braces of (B8) equals $-\left(d^{2}-d\right.$ +1 ), which is the prefactor of $\delta_{k \|} \delta_{l \|}$ in (B3) for $y=d-1$, and thus (B8) is consistent with (B2) and (B6). The amplitude $S$ follows from (B4) and (B9) with the Gaussian ${ }^{17}$ value $B_{T}$ $=[\Gamma(d / 2)]^{2} d /\left[4 \pi^{d}(d-1)\right]$, and the expression (3.33) then reproduces the form (3.31) of the needle coefficient $\mathcal{N}$.

Finally consider the profile of the energy density or $\psi$. No contribution comes from the $T_{\|\|}$integral in (3.32). The contribution of order $R^{2\left(x_{\epsilon}-1\right)}$ follows from Eq. (2.2) and the $\mathrm{e}^{17}$ form $C_{\psi \psi \psi \psi}\left(r_{12} r_{13} r_{23}\right)^{-x_{\epsilon}}$ of the bulk three-point function $\left\langle\psi\left(\mathbf{r}_{1}\right) \psi\left(\mathbf{r}_{2}\right) \psi\left(\mathbf{r}_{3}\right)\right\rangle_{\text {bulk }}$ as

$$
\left.\langle\psi(\mathbf{r})\rangle_{\mathrm{cyl}}\right|_{\propto R^{2\left(x_{\epsilon}-1\right)}}=-K C_{\psi \psi \psi} R^{2\left(x_{\epsilon}-1\right)} r_{\perp}^{-\left(3 x_{\epsilon}-2\right)} D\left(x_{\epsilon}\right) .
$$

Here

$$
\begin{aligned}
D(x)= & 2^{2(1-x)} \pi \Gamma((1-x) / 2) \Gamma((3 x / 2)-1) \\
& \times \Gamma(x-(1 / 2)) /\left\{[\Gamma(x / 2)]^{2} \Gamma(x)\right\}
\end{aligned}
$$

for $x>1$ is the analytic continuation from $2 / 3<x<1$ of

$$
\int_{-\infty}^{\infty} \mathrm{d} \rho_{1} \int_{-\infty}^{\infty} \mathrm{d} \rho_{2}\left|\rho_{1}-\rho_{2}\right|^{-x}\left[\left(1+\rho_{1}^{2}\right)\left(1+\rho_{2}^{2}\right)\right]^{-x / 2} .
$$

For the Gaussian model where $K$ is given in (3.28), $C_{\psi \psi \psi}$ $=2^{3(2-d)}$, and $\psi$ is related to $\Phi^{2}$ as below (A18), Eqs. (B10) and (B11) are consistent with the expression of order $R^{4 \alpha}$ for $\left\langle\Phi^{2}(\mathbf{r})\right\rangle_{\text {cyl }}$ that follows from the propagator $\langle\varphi\rangle_{\text {cyl }}$ in (3.25) as $\mathbf{r}^{\prime} \rightarrow \mathbf{r}$.

${ }^{1}$ Soft Matter, edited by G. Gompper and M. Schick (Wiley-VCH, Weinheim, 2005).

${ }^{2}$ Polymer depletion interactions between a pair of immersed particles and between a single particle and a wall have been measured in recent experiments: R. Verma, J. C. Crocker, T. C. Lubensky, and A. G. Yodh, Macromolecules 33, 177 (2000); D. Rudhardt, C. Bechinger, and P. Leiderer, J. Phys.: Condens. Matter 11, 10073 (1999); D. Kleshchanok, R. Tuinier, and P. R. Lang, Langmuir 22, 9121 (2006).

${ }^{3}$ E. Eisenriegler, in Soft Matter, edited by G. Gompper and M. Schick (Wiley-VCH, Weinheim, 2005), Vol. 2; Lecture Notes in Physics, edited by H. Meyer-Ortmanns and A. Klümper (Springer, Berlin 1998), Vol. 508, pp. 1-24.

${ }^{4}$ P. G. de Gennes, Scaling Concepts in Polymer Physics (Cornell University, Ithaca, 1979).

${ }^{5}$ E. Eisenriegler, A. Hanke, and S. Dietrich, J. Chem. Phys. 54, 1134 (1996); A. Hanke, E. Eisenriegler, and S. Dietrich, Phys. Rev. E 59, 6853 (1999). The dimension $d_{\perp}=d-1$ of the space perpendicular to the particle axis is denoted by $d$ in these articles.

${ }^{6}$ For ideal polymers in three dimensions, $m-1=0$, and a cylinder or needle is a "marginal" perturbation with a free energy of immersion that vanishes like $1 / \ln (1 /$ width $)$ as the width $2 R$ or $s$ tends to zero. The cylinderneedle relations (2.9) and (2.10) do not apply to this case.

${ }^{7}$ A similar difference arises between needle and disk.

${ }^{8}$ Ellipsoids of arbitrary aspect ratio $s / l$ interacting with ideal polymers in $d=3$ are discussed in E. Eisenriegler, A. Bringer, and R. Maassen, J. Chem. Phys. 118, 8093 (2003) and in the first review in Ref. 3. See also T. Odijk, Physica A 278, 347 (2000).

${ }^{9}$ P. G. de Gennes, C. R. Seances Acad. Sci., Ser. B 288, 359 (1979).

${ }^{10}$ E. Eisenriegler, J. Chem. Phys. 124, 144912 (2006).

${ }^{11}$ T. W. Burkhardt and J.-Y. Choi, Nucl. Phys. B 376, 447 (1992). This paper discusses the marginal perturbation of a defect line in the twodimensional Ising model where $x_{\epsilon}-1=0$.

${ }^{12} \mathrm{~K}$. Binder, in Phase Transitions and Critical Phenomena, edited by C. Domb and J. L. Lebowitz (Academic, London, 1983), Vol. 8, p. 1; H. W. Diehl, in Phase Transitions and Critical Phenomena, edited by C. Domb and J. L. Lebowitz (Academic, London, 1986), Vol. 10, p. 75; H. W. Diehl, Int. J. Mod. Phys. B 11, 3503 (1997).

${ }^{13}$ In terms of the unit vector $\boldsymbol{\rho}$ along the particle axis introduced in Ref. 10, $T_{\|\|}=\Sigma_{k l} \rho_{k} \rho_{l} T_{k l}$ and $\partial_{\|}=\Sigma \rho_{k} \partial_{k}$.

${ }^{14} \mathrm{We}$ always consider the scaling limit where polymer and particle sizes are much larger than the microscopic lengths.

${ }^{15}$ In the polymer limit $n \rightarrow 0$ the amplitude $J$ in the leading term of the cylinder expansion (2.2) is related by $J=2^{m} A_{d-1, d} / A_{d, d}$ to the amplitudes of the "generalized cylinder" introduced in Refs. 5. Here $A_{d, d}$ equals the sphere amplitude $A$ defined below Eq. (4.4). For ideal (Refs. 5) polymers $A_{d_{\perp}, d}=2 \pi^{d_{\perp} / 2} / \Gamma\left(\left(d_{\perp}-2\right) / 2\right)$ is consistent with the result (3.28) for $J$. For self-avoiding polymers in $d=3$, the estimates $A_{2,3} \approx 1.23$ and $A_{3,3} \approx 9.82$ in the second article in Ref. 5 yield $J \approx 0.308$.

${ }^{16}$ For example, an $R$-derivative term $\propto \int \mathrm{d} r_{\|}\left(\partial R / \partial r_{\|}\right)^{2} R^{x_{\epsilon}-1} \psi$ in (2.2) yields contributions to $\mathcal{I}$ and $\mathcal{N}^{\prime}$ which are smaller by a factor $(s / l)^{2}$ than the leading expressions in Eq. (2.9).

${ }^{17}$ E. Eisenriegler, J. Chem. Phys. 121, 3299 (2004).

${ }^{18}$ Equation (3.29) follows from Eq. (C7) in Ref. 17 on replacing $\epsilon \rightarrow-\psi$, so that $B_{\epsilon} \rightarrow \mathcal{A}_{\epsilon}^{2}$, and on using the Gaussian value of $\mathcal{A}_{\epsilon}$ given below (A18). We correct three misprints in Ref. 17: in (C4) the equation $C_{\varphi \varphi \epsilon} /\left(B_{\varphi} B_{\epsilon}^{1 / 2}\right)=\sqrt{2}$ should be replaced by $C_{\varphi \varphi \epsilon} /\left(B_{\varphi} B_{\epsilon}^{1 / 2}\right)=-\sqrt{2}$, in $(\mathrm{C} 7)$ the equation $C_{\epsilon \epsilon \epsilon} / B_{\epsilon}^{3 / 2}=2^{3 / 2}$ should be replaced by $C_{\epsilon \epsilon \epsilon} / B_{\epsilon}^{3 / 2}=-2^{3 / 2}$, and above Eq. (4.11) the expression $\left(\partial_{\| P}\right) \varphi+\varphi\left(\partial_{\| Q}\right)$ should be replaced by $\left(\partial_{\| P}^{2} \varphi\right) \varphi+\varphi\left(\partial_{\| Q}^{2} \varphi\right)$.

${ }^{19} \mathrm{We}$ expect additional terms in the cylinder expansion such as $\propto$ $-R^{x^{+}+1} \int \mathrm{d} r_{\|} \Delta_{\mathbf{r}} \psi$. In the Gaussian model the exponent of $R$ here is also $d$ -1 . However, this term does not contribute to $\mathcal{N}$ in the ellipsoid expansion.

${ }^{20} \delta F_{\text {ori }}$ and $\delta F_{\text {ori }}^{\prime}$ correspond to the quantities $\delta F_{\text {aniso }}$ and $\delta F_{\text {aniso }}^{\text {(next) }}$ in Ref. 10.

${ }^{21}$ For a small sphere $\mathcal{I}=\left(2 R_{\text {sphere }}\right)^{m}$.

${ }^{22}$ For ideal chains $A=2 \pi^{d / 2} / \Gamma((d-2) / 2), B=2$. Using Eqs. (3.23) and (3.24) and the results at the end of Appendix A, one finds that for a needle $\mathcal{N}^{\prime} / \mathcal{N}$ is proportional to $(l / s)^{d-3}$ if $d>3$ and equals $[\ln (2 l / s)$ $-3 / 4] /(2 \pi)$ if $d=3$.

${ }^{23}$ This term corresponds to the $\mathcal{N}$ contribution in (2.1). The term $\propto l^{3.30} z^{-0.30}$ corresponding to the $\mathcal{N}^{\prime}$ contribution is negligible both (Ref. 10) for the disk and for ellipsoids with aspect ratios $s / l$ of order 1.

${ }^{24}$ Compare, e.g., C. Flammer, Spheroidal Wave Functions (Stanford University Press, Stanford, 1957).

${ }^{25} S_{\beta, \gamma}(\eta)$ satisfies the differential equation (A4) with $\xi$ replaced by $\eta$ and remains finite for $\eta \rightarrow \pm 1$. Note that $-1<\eta<1$ while $1<\xi<\infty$.

${ }^{26} R_{0, \gamma}^{(\infty)}(\xi \rightarrow 1)$ has a logarithmic divergence (Refs. 6 and 8 ) in $d=3$ and is finite in $2<d<3$.

${ }^{27}$ The small ellipsoid should be distinguished from the limit of the cylinder, where $l \rightarrow \infty$ for fixed $s=2 R$ and $r_{\perp}$ and where Eqs. (3.7), (3.8), and (3.10) imply $\xi-1 \rightarrow 2\left(r_{\perp} / l\right)^{2}$ and $\xi_{0}-1 \rightarrow(s / l)^{2} / 2$, respectively. For example, consider the end density in (3.15) and (3.16) in the cylinder limit. Since for $\xi \backslash 1$ and $d>3, \kappa(\xi)$ tends to $4 /\left[(d-3)(\xi-1)^{(d-3) / 2}\right]$, the end density $e(\mathbf{r})$ becomes $1-\left(R / r_{\perp}\right)^{d_{\perp}-2}$, where $d_{\perp}=d-1$. Thus for a cylinder in $d$ dimensions the $r_{\perp}$ dependence of $e$ is the same as the $r$ dependence for a sphere in $d-1$ dimensions. This is expected since the ideal chain partition function factors into contributions perpendicular and parallel to the cylinder axis.

${ }^{28}$ At the critical point the bulk two-point function $\left\langle T_{k l} \psi\right\rangle_{\text {bulk }}$ vanishes while the three-point function $\left\langle T_{k l} \psi\left(\mathbf{r}_{1}\right) \psi\left(\mathbf{r}_{2}\right)\right\rangle_{\text {bulk }}$ is nonvanishing.

${ }^{29}$ See L. S. Brown, Ann. Phys. (N.Y.) 126, 135 (1980) or Eqs. (1.15) in E. Eisenriegler, M. Krech, and S. Dietrich, Phys. Rev. B 53, 14377 (1996).

${ }^{30}$ The integrals on the right hand side of (B6) on substituting (B5) are all of the type 6.576.4 in Gradsteyn and Ryshik. 